\title{
Systematic literature review and evaluation of patient reported outcome measures (PROMs) for asthma and related allergic diseases
}

\author{
Allison Worth', *Victoria S Hammersley', Ulugbek Nurmatov', Aziz Sheikh' \\ ${ }^{1}$ Allergy and Respiratory Research Group, Centre for Population Health Sciences, The University of Edinburgh, Medical School, Edinburgh, UK
} Originally received 22nd May 2012; resubmitted 22nd June 2012; revised 29th June 2012; accepted 2nd July 2012; online 21st September 2012

(C) 2012 Primary Care Respiratory Society UK. All rights reserved.

A Worth et al. Prim Care Respir J 2012; 21(4): 455-458

http://dx.doi.org/10.4104/pcrj.2012.00084

Keywords allergy, asthma, patient reported outcome measures

The full version of this paper, with online appendix,

is available online at www.thepcrj.org

\section{Background}

Patient reported outcome measures (PROMs) are measures of the outcome of treatment that are reported directly by the patient or carer. ${ }^{1}$ Such outcomes may include symptoms, well-being, health/functional status, health-related quality of life (HRQL), satisfaction with treatment and outcomes, and perceptions of the humanity of care. ${ }^{1-3}$ PROMs are typically short self-completed questionnaires, most commonly used to measure patients' health status or HRQL before and after an intervention. . ${ }^{4}$ Over 3,000 generic and disease-specific PROMs exist, ${ }^{5}$ and these are now commonly used in research contexts, particularly clinical trials.

From April 2009 the National Health Service (NHS) became the first health system in the world to advocate routinely collecting PROMs. ${ }^{6}$ High Quality Care for All emphasised the need for the NHS to assess effectiveness of care from the patient's perspective using PROMs. PROMs data could potentially contribute to determinants of service quality so that patient assessments of the quality of their experiences could be compared across services and between providers and have an impact on NHS funding ${ }^{8}$ and patient choice. PROMs are currently being used to collect patient perceptions of the quality of care they receive from the NHS in England in relation to hip and knee replacements, hernia repair and varicose vein surgery, with pilot work beginning into their use for long-term conditions including asthma and chronic obstructive pulmonary disease.

The use of PROMs in clinical settings as part of day-to-day practice with the purpose of demonstrating improved health and supporting clinical decision-making raises a number of challenges.
Unresolved issues include; ${ }^{3,6,8-13}$

- which instruments to choose;

- when and how to administer these;

- how to promote their appropriate use and standardisation in trials and clinical practice;

- potential clinician and patient questionnaire fatigue;

- the problem of assessing co-morbidities (i.e. patients may need to complete several disease-specific measures and a generic measure);

- the difficulties in isolating provider effects from other factors which can influence outcomes;

- the lack of validation of non-English language versions of PROMs;

- the role of proxy-reported outcome measures;

- ensuring adequate response rates.

Furthermore, mechanisms are unclear for adjusting raw data to account for variation in patient characteristics to allow meaningful comparisons between care settings, sites, providing feedback to clinicians and patients, and how the data will be used to improve the quality of care. ${ }^{6}$ There is therefore a need to undertake considerable developmental work before PROMs can be rolled out to the management of people with long-term conditions. ${ }^{6}$ This includes a crucial need for methodological work on validation of PROMs to ensure responsiveness, construct validity, reliability, internal consistency, and acceptability.

Clinicians, managers, and policy makers need to be able to interpret the meaning of scores if PROMs are to be used routinely as evidence for decision-making, as statistical and clinical relevance may differ. ${ }^{14}$ An important issue for policy makers is how PROMs can aid policy decisions in allocating healthcare resources. ${ }^{10}$ Aside from readily quantifiable data such as the length of waiting lists or

\footnotetext{
* Corresponding author: Ms Victoria S Hammersley, Allergy and Respiratory Research Group, Centre for Population Health Sciences, The University of Edinburgh, Medical School, Teviot Place, Edinburgh EH8 9AG, UK. Tel: +44 (0)131 6503234 E-mail: vicky.hammersley@ed.ac.uk
} 
complication rates, there is a distinct need for valid outcome measures related to patient satisfaction. However, patient satisfaction is a complex entity which consists of elements such as personal affinity with caregivers which may be unduly emphasised if patients are asked to assess their medical care by simple enquiry. More important in this regard is the need to establish true medical benefit and improvement in quality of life from the patient's point of view, hence PROMs must be selected which can be validated for this purpose.

Patient perspectives of PROMs are also important and underevaluated, and the proposed uses and benefits of completing PROMs should be transparent. It does appear that patients find PROMs acceptable in terms of time and effort: research by members of our team, administering HRQL questionnaires in an outpatient allergy clinic setting, identified very high response rates in children (73-94\%) and adults (80-86\%) and little questionnaire fatigue. ${ }^{15-17}$ The intended use of PROMs by patients to inform effective choices in health care services requires further examination, ${ }^{10,13}$ particularly in relation to informed decision-making and health literacy. ${ }^{18}$

Asthma and related allergic disorders affect up to one in three of the UK population at some point in their lives, and are responsible for considerable morbidity and in some cases mortality. ${ }^{19}$ The quality of allergy services is under government scrutiny and there is thus a pressing need to assess the impact of care provision from the perspective of patients and their carers. Allergic diseases can have a considerable impact on personal health and well-being across the age spectrum and have a substantial impact on health services in primary, secondary, and tertiary care. The direct costs to the NHS have been estimated to be well in excess of $£ 1$ billion per annum..$^{20,21}$ Patients with allergic disease often experience other allergic comorbidities, so the use of PROMs for multiple conditions can be explored with this group. Allergic diseases are therefore good exemplar long-term conditions through which to examine methodological development in extending the use of PROMs to a wider patient population.

The House of Lords Allergy Inquiry ${ }^{22}$ identified a number of concerns regarding outcome measures in allergy: the vast array of outcome measures used in research settings; their specificity (i.e. related to one condition rather than the whole person); gaps in relation to allergic conditions other than asthma, eczema and rhinitis; and the fact that they do not always include issues that are pertinent to patients. The proposed research will identify currently available PROMs for use by patients with allergic disease and make recommendations on whether - and, if so, how - these can be adapted for use in clinical and research settings. There are wellestablished PROMs for eczema, asthma, and allergic rhinitis, urticaria, food and venom allergy: $i^{15-17,23-29}$ a range of other less wellknown measures for various allergic conditions; $; 0,31$ and generic measures such as the SF36 and EQ5D ${ }^{32,33}$ are also used. We will build on existing work in this field. We are aware of ongoing Department of Health pilot work on PROMs for asthma (http://www.publichealth.ox.ac.uk/research/hsru/promspilot/project outline) and intend our work to complement and extend this work for other allergic conditions. We anticipate there will be few, if any, validated instruments for drug allergy, anaphylaxis, and angiooedema. We will therefore also look at the use of generic PROMs, identify research gaps, and address development, validation, and quality issues for PROMs for these specific conditions.

\section{Objectives}

- To identify all available validated generic and disease-specific PROMs for asthma and related allergic conditions in adults and children (i.e. articles where the PROM was published first and its development described).

- To identify all relevant methodological work reporting on validation of the disease-specific PROMs.

- To examine the validity of each disease-specific PROM identified.

- To identify clinicians' perspectives regarding the use of PROMs in clinical contexts.

- To identify gaps in the PROMs available for asthma and related allergic conditions (including co-existent conditions) and groups of patients/carers.

- To describe which generic PROMs are used in conjunction with disease-specific PROMs identified.

\section{Review methodology}

\section{Search strategy}

The following databases will be searched for relevant studies (see Appendix 1, available online at www.thepcrj.org, for search strategies)

- MEDLINE

- EMBASE

- Web of Science: Science Citation Index, Conference Proceedings Citation Index- Science

The following websites will be searched for relevant studies:

- PROQOLID (http://www.proqolid.org)

- PROMIS (http://www.nihpromis.org)

- American Thoracic Society QOL resource (http://qol.thoracic.org/)

Searches will be limited to literature from 1990 onwards; this limit is based on the first publication of key PROMs such as the Rhinoconjunctivitis Quality of Life Questionnaire and Asthma Quality of Life Questionnaire. ${ }^{24,34}$ Additional references will be located through searching the references cited by the identified studies, and unpublished work and research in progress will be identified through discussion with experts in the field and searching the National Institute of Health Research and Agency for Healthcare Research and Quality databases and Google Scholar. We will invite experts who are active in the field from a range of disciplines and geography to comment on our search strategy and the list of included studies. There will be no language restrictions and, where possible, all literature will be translated. We will report any literature which we are unable to translate. We will report our findings using the PRISMA checklist. ${ }^{35}$

\section{Inclusion criteria}

- The PROM must be specifically designed for use in patients/carers with any of the following allergic conditions: atopic dermatitis/eczema, food allergy, asthma, allergic rhinitis 
and/or conjunctivitis, urticaria, angio-oedema, venom allergy, drug allergy, oral allergy syndrome, and anaphylaxis.

- Any articles relating to the description, development and/or the validation of identified PROMs.

- The identified PROM must be available for use in the English language.

Exclusion criteria

- Reviews, letters and editorials.

- Economic theory.

- Clinician-based outcome measures.

Review strategy

The titles will be checked independently by two reviewers according to the above selection criteria and categorised as included, not included, and unsure. For those papers in the unsure category we will retrieve the abstract and re-categorise as above. Any discrepancies will be resolved by consensus and, if necessary, a third reviewer will be consulted. Full text copies of potentially relevant studies will be obtained and their eligibility for inclusion independently assessed. Studies that do not fulfil all of the inclusion criteria will be excluded. Quality assessment and data extraction will be conducted by two reviewers independently, with arbitration where necessary.

\section{Quality assessment strategy}

The development and performance of any outcome measurement should be rigorously tested before it can be used in a clinical setting or as a research tool. ${ }^{36} \mathrm{We}$ propose to assess the development of the instruments identified and their performance properties including validity, generalisability, responsiveness, managing missing data, how the significance of results is interpreted, what minimum reported difference is considered relevant, how variation in patient demography is managed, cross-cultural and linguistic adaptation using a previously reported quality assessment tool. ${ }^{37}$

A team of researchers will independently assess the articles against the defined criteria and any discrepancies will be resolved by consensus and, if necessary, a third reviewer will be consulted.

\section{Analysis and data synthesis}

The properties of the various instruments (outcomes measures) identified will be described in detail and a narrative synthesis will be presented summarising the development and validation of each identified outcome measure. We will assess the clinical utility of the outcome measures identified and report on the extent to which tools are fit for clinical and research purposes. Clinical and statistical significance of each outcome measure will be reported where available with a view to identifying which tools, if any, are ready for clinical use and which need further work. From the literature identified by the systematic review, we will report any additional use of the outcome measures (e.g. commissioning or performance management). We will also report on any patient and clinician perceptions of the impact of PROMs in clinical settings.

\section{Handling editor Niels Chavannes}

Acknowledgements The following people form our Project Management Group and have contributed to the development of the protocol: Professor Anthony Dubois, Dr Samantha Walker, Dr Rebecca Knibb, Dr Bertine Flokstra de-Blok, Dr Audrey DunnGalvin.
Conflicts of interest The authors declare that they have no conflicts of interest in relation to this protocol. AS is Joint Editor-in-Chief of the $P C R J$, but was not involved in the editorial review of, nor the decision to publish, this article.

Contributorship AS and AW conceived the study. UN wrote the search strategies. AW and VH drafted the manuscript and all authors commented on draft versions, and read and approved the final manuscript.

Funding The proposed systematic review has been funded by NAPP Pharmaceuticals Limited.

\section{References}

1. Smith S, Cano S, Lamping D, et al. Patient-Reported Outcome Measures (PROMs) for routine use in treatment centres: recommendations based on a review of the scientific evidence. London: Health Services Research Unit, London School of Hygiene and Tropical Medicine, 2005.

2. Bradley C. Feedback on the FDA's February 2006 draft guidance on Patient Reported Outcome (PRO) measures from a developer of PRO measures. Health and Quality of Life Outcomes 2006;4:78. http://dx.doi.org/10.1186/1477-7525-4-78

3. Black $N$, Jenkinson C. Measuring patients' experiences and outcomes. BMJ 2009;339:b2495. http://dx.doi.org/10.1136/bmj.b2495

4. Department of Health (DH). Guidance on the routine collection of Patient Reported Outcome Measures (PROMs). London: DH, 2008.

5. Medical Research Council (MRC). Patient Reported Outcome Measures (PROMs): identifying UK research priorities. London: MRC, 2009.

6. Appleby J. PROMs: Counting what matters most to patients. In: The King's Fund Blog, 9 February 2009

7. Department of Health (DH). High Quality Care for All: NHS next stage review final report. London: $\mathrm{DH}, 2008$.

8. Devlin N, Parkin D, Browne J. Using the EQ-5D as a performance measurement tool in the NHS. Discussion paper 09/03. London: Department of Economics, City University, 2009.

9. Greenhalgh J. The application of PROs in clinical practice: what are they, do they work and why? Qual Life Res 2009;18:115-23. http://dx.doi.org/10.1007/s11136-008-9430-6

10. Devlin N, Appleby J. Getting the most out of PROMs. Putting health outcomes at the heart of NHS decision-making. London: The King's Fund, 2010.

11. Dawson J, Doll H, Fitzpatrick R, Jenkinson C, Carr AJ. The routine use of patient reported outcome measures in healthcare settings. BMJ 2010;340:c186. http://dx.doi.org/10.1136/bmj.c186

12. Browne J, Jamieson L, Lawsey J, et al. Patient Reported Outcome Measures (PROMs) in elective surgery. Report to the Department of Health. London: Health Services Research Unit, London School of Hygiene and Tropical Medicine, 2007.

13. Appleby J, Devlin N. Measuring success in the NHS. Using patient-assessed health outcomes to manage performance of healthcare providers. London: The King's Fund, 2004.

14. Redelmeier D, Guyatt G, Goldstein R. Assessing the minimal important difference in symptoms: a comparison of two techniques. J Clin Epidemiol 1996;49(11):12159. http://dx.doi.org/10.1016/S0895-4356(96)00206-5

15. Flokstra-de Blok BM, DunnGalvin A, Vlieg-Boerstra BJ, et al. Development and validation of the self-administered Food Allergy Quality of Life Questionnaire for adolescents. J Allergy Clin Immunol 2008;122(1):139-44. http://dx.doi.org/10.1016/j.jaci.2008.05.008

16. Flokstra-de Blok BM, DunnGalvin A, Vlieg-Boerstra BJ, et al. Development and validation of a self-administered Food Allergy Quality of Life Questionnaire for children. Clin Exp Allergy 2009;39(1):127-37 http://dx.doi.org/10.1111/j.1365-2222.2008.03120.x

17. Flokstra-de Blok BM, van der Meulen GN, Dunngalvin A, et al. Development and validation of the Food Allergy Quality of Life Questionnaire-Adult Form (FAQLQAF). Allergy 2009;64:1209-17. http://dx.doi.org/10.1111/j.1398-9995.2009. 01968.x

18. Coulter A, Ellins J. Effectiveness of strategies for informing, educating and involving patients. BMJ 2007;335:24-7 http://dx.doi.org/10.1136/bmj.39246.581169.80

19. Scottish Medical and Scientific Advisory Committee. Review of Allergy Services in Scotland. Edinburgh: Scottish Government Directorates, 2009.

20. Gupta R, Sheikh A, Strachan DP, Anderson HR. Burden of allergic disease in the UK: secondary analyses of national databases. Clin Exp Allergy 2004;34(4):520-6. 
http://dx.doi.org/10.1111/j.1365-2222.2004.1935.x

21. Anandan C, Gupta R, Simpson CR, Fischbacher C, Sheikh A. Epidemiology and disease burden from allergic disease in Scotland: analyses of national databases. J R Soc Med 2009;102(10):431-42. http://dx. doi.org/10.1258/jrsm.2009.090027

22. House of Lords Science and Technology Committee. Allergy. 6th report of session 2006-2007. London: The Stationery Office, 2007.

23. Lewis-Jones MS, Finlay AY, Dykes PJ. The infants' dermatitis quality of life index. $\mathrm{Br}$ J Dermatol 2001;144(1):104-10. http://dx.doi.org/10.1046/j.1365-2133.2001.03960.x

24. Juniper EF, Guyatt GH, Epstein RS, Ferrie PJ, Jaeschke R, Hiller TK. Evaluation of impairment of health related quality of life in asthma: development of a questionnaire for use in clinical trials. Thorax 1992;47(2):76-83. http://dx.doi.org/10.1136/thx.47.2.76

25. Juniper $E$, Riis $B$, Juniper $B$. Development and validation of an electronic version of the Rhinoconjunctivitis Quality of life Questionnaire. Allergy 2007;62:1091-3. http://dx.doi.org/10.1111/j.1398-9995.2007.01370.x

26. Elberink JN, De Monchy JG, Golden D, Brouwer J-L, Guyatt GH, Dubois AE. Development and validation of a health-related quality-of-life questionnaire in patients with yellow jacket allergy. J Allergy Clin Immunol 2002;109:162-70. http://dx.doi.org/10.1067/mai.2002.120552

27. Charman C, Venn A, Williams H. The Patient-Oriented Eczema Measure. Development and initial validation of a new tool for measuring atopic eczema severity from the patients' perspective. Arch Dermatol 2004;140:1513-19. http://dx.doi.org/10.1001/archderm.140.12.1513

28. Basra MK, Fenech R, Gatt RM, Salek MS, Finlay AY. The Dermatology Life Quality Index 1994-2007: a comprehensive review of validation data and clinical results. $\mathrm{Br}$ J Dermatol 2008;159(5):997-1035.
29. Hanifin JM, Thurston M, Omoto M, Cherill R, Tofte SJ, Graeber M. The eczema area and severity index (EASI): assessment of reliability in atopic dermatitis. Exp Dermatol 2001;10:11-18. http://dx.doi.org/10.1034/j.1600-0625.2001.100102.x

30. Roberts G, Hurley C, Lack G. Development of a quality-of-life assessment for the allergic child or teenager with multisystem allergic disease. J Allergy Clin Immunol 2003;111(3):491-7. http://dx.doi.org/10.1067/mai.2003.138

31. Baiardini I, Pasquali M, Braido F, et al. A new tool to evaluate the impact of chronic urticaria on quality of life: Chronic Urticaria Quality of Life Questionnaire (CU-QoL). Allergy 2005;60(8):1073-8. http://dx.doi.org/10.1111/j.1398-9995.2005.00833.x

32. Ware JE Jr, Sherbourne CD. The MOS 36-item short-form health survey (SF-36). I. Conceptual framework and item selection. Med Care 1992;30(6):473-83. http://dx.doi.org/10.1097/00005650-199206000-00002

33. EuroQol Group. EuroQol - a new facility for the measurement of health-related quality of life. Health Policy 1990;16:199-208. http://dx.doi.org/10.1016/0168-8510(90)90421-9

34. Juniper E, Guyatt GH. Development and testing of a new measure of health status for clinical trials in rhinoconjunctivitis. Clin Exp Allergy 1991;21:77-83. http://dx.doi.org/10.1111/j.1365-2222.1991.tb00807.x

35. Moher D, Liberati A, Tetzlaff J, Altman DG, Prisma Group. Preferred reporting items for systematic reviews and meta-analyses: the PRISMA statement. PLoS Med/Public Library of Science 2009;6(7):e1000097.

36. Fitzpatrick R, Davey C, Buxton M, Jones D. Evaluating patient-based outcome measures for use in clinical trials. Health Technol Assess 1998;2(14).

37. Pesudovs K, Burr JM, Harley C, Elliott DB. The development, assessment, and selection of questionnaires. Optom Vis Sci 2007;84(8):663-74. http://dx.doi.org/10.1097/OPX.0b013e318141fe75

\section{Available online at http://www.thepcrj.org}




\section{Web of Science: Science Citation Index, Conference Proceedings Citation Index- Science, 1990- present}

(Hypersensitivity or allergy or atopy or atopic dermatitis or eczema or food allergy or asthma or rhinitis or rhinoconjunctivitis or urticaria or angioedema or venom allergy or drug allergy or oral allergy syndrome or anaphylaxis)

\section{AND}

(PROMs or PROM or patient reported outcome measure* or questionnaire* or HRQL or quality of life or health related quality of life or patient satisfaction or consumer satisfaction or patient preference or patient participation or "patient acceptance of healthcare" or patient outcome or patient based outcome or functional status or health status or subjective health status or health status indicator or health status assessment)

\section{AND}

(methodol* or psychometric* or validity or reliability or responsiveness or effect size or sensitivity to change or reproducibility or acceptability or utility measure*)

\section{EMBASE 1990-present}

1. (PROMs or PROM).mp. [mp=title, abstract, subject headings, heading word, drug trade name, original title, device manufacturer, drug manufacturer, device trade name, keyword]

2. Health Related Quality of Life.mp.

3. HRQL.mp.

4. Health Status/

5. subjective health status.mp.

6. "Quality of Life".mp.

7. "outcome assessment (health care)".mp.

8. patient reported outcome measure*.mp.

9. patient outcome.mp.

10. patient based outcome.mp.

11. patient preference.mp.

12. Patient Participation/

13. patient participation.mp.

14. "patient acceptance of health care".mp.

15. consumer satisfaction.mp.

16. Patient Satisfaction/

17. patient satisfaction.mp.

18. health status indicator*.mp.

19. or/1-18

20. methodol*.mp.

21. psychometric*.mp.

22. validity.mp.

23. reliability.mp.
24. responsiveness.mp.

25. effect size.mp.

26. sensitivity to change.mp.

27. reproducibility.mp.

28. acceptability.mp.

29. utility measure*.mp.

30. or/20-29

31. 19 and 30

32. Hypersensitivity/

33. predisposition.mp.

34. allerg*.mp.

35. react*, allergic.mp.

36. reaction, allerg*.mp.

37. atop*.mp.

38. or/32-37

39. Dermatitis, Atopic/

40. dermatitis.mp.

41. dermatitides.mp.

42. atopic dermatitis.mp.

43. dermatitis, atopic.mp.

44. itching.mp.

45. or/39-44

46. Eczema/

47. eczema.mp.

48. atopic eczema.mp.

49. eczematous dermatiti*.mp.

50. dermatiti*, eczematous.mp.

51. eczema, atopic.mp.

52. or/46-51

53. food allerg*.mp.

54. food hypersensitivity.mp.

55. food hypersensitivities.mp.

56. allergy, food.mp.

57. oral allergy syndrome.mp.

58. or/53-57

59. exp Asthma/

60. asthma.mp. or Asthma/

61. asthmatic children.mp.

62. acute asthmatic attack.mp.

63. night cough*.mp.

64. wheez*.mp.

65. bronchial disorder.mp.

66. hyper-responsiveness wheez*.mp.

67. lung function.mp.

68. ventilatory function.mp.

69. FEV.mp.

70. FEF.mp.

71. FVC.mp.

72. PEF.mp.

73. bronchial hyperreactivity.mp.

74. airway hyperreactivity.mp.

75. bronchial responsiveness.mp.

76. airway responsiveness.mp. 
Appendix 1. Details of search strategy continued

77. or/59-76

78. Rhinitis, Allergic, Perennial/

79. Rhinitis, Allergic Seasonal/

80. hayfever.mp.

81. hay fever.mp.

82. fever, hay.mp.

83. seasonal allergic rhinitis.mp.

84. allergic rhinitides. $\mathrm{mp}$.

85. allergic rhinitis.mp.

86. rhiniti*.mp.

87. poll?nosis.mp.

88. pollenosis.mp.

89. Conjunctivitis, Allergic/

90. conjunctivit*.mp.

91. rhino-conjunctivit*.mp.

92. or/78-91

93. Urticaria/

94. urticaria.mp.

95. angioedema.mp.

96. or/93-95

97. venom allergy.mp.

98. Drug Hypersensitivity/

99. drug allergy.mp.

100. or/97-99

101. Anaphylaxis/

102. anaphylaxis react*.mp.

103. anaphylactic react* ${ }^{*}$.

104. anaphylactic shock*.mp.

105. anaphylactoid syndrome*.mp.

106. anaphylactoid react*.mp.

107. anaphylactic syndrome*.mp.

108. anaphylactoid shock*.mp.

109. acute systemic allergic react*.mp.

110. idiopathic anaphylaxis.mp.

111. systemic anaphylaxis.mp.

112. or/101-111

113. 38 or 45 or 52 or 58 or 77 or 92 or 96 or 100 or 112

114. 31 and 113

115. animals/ not (humans/ not animals/)

116. 113 not 115

117. limit 116 to $y r=" 1990$-Current"

\section{MEDLINE 1990-present}

1. PROMs.or PROM.mp.

2. "Quality of Life" /

3. Health Related Quality of Life.mp.

4. HRQL.mp.

5. Health Status/

6. Subjective health status.mp.

7. "Outcome Assessment (Health Care)"/
8. "outcome assessment (health care)".mp.

9. patient reported outcome measure*.mp.

10. patient outcome.mp.

11. "Outcome and Process Assessment (Health Care)"/

12. patient based outcome.mp.

13. Patient Preference/

14. patient preference.mp.

15. Patient Participation/

16. patient participation.mp.

17. "Patient Acceptance of Health Care"/

18. "patient acceptance of health care".mp.

19. Consumer Satisfaction/

20. consumer satisfaction.mp.

21. Patient Satisfaction/

22. patient satisfaction.mp.

23. Health Status Indicators/

24. health status indicator*.mp.

25. or/1-24

26. Methodol*.mp.

27. Psychometric*.mp.

28. Validity.mp.

29. Reliability.mp.

30. Responsiveness.mp.

31. Effect size.mp.

32. Sensitivity to change.mp.

33. Reproducibility.mp.

34. Acceptability.mp.

35. Utility measure*

36. or/26-35

37. 25 and 36

38. exp Hypersensitivity/

39. predisposition.mp.

40. allerg*.mp.

41. react*, allergic.mp.

42. reaction, allerg*.mp.

43. atop*.mp

44. or/38-43

45. Dermatitis, Atopic/

46. dermatitis.mp.

47. dermatitides.mp.

48. atopic dermatitis.mp.

49. dermatitis, atopic.mp.

50. itching.mp.

51. or/45-50

52. Eczema/

53. eczema.mp.

54. atopic eczema.mp.

55. eczematous dermatiti*.mp. 
Appendix 1. Details of search strategy continued

56. dermatiti*, eczematous.mp.

57. eczema, atopic.mp

58. or/52-57

59. Food Hypersensitivity/

60. food allerg*.mp.

61. food hypersensitivity.mp.

62. food hypersensitivities.mp.

63. allergy, food.mp.

64. oral allergy syndrome.mp.

65. or/59-64

66. $\exp$ Asthma/

67. asthma.mp. or Asthma/

68. asthmatic children.mp.

69. acute asthmatic attack.mp.

70. night cough*.mp.

71. wheez*.mp.

72. Respiratory Hypersensitivity/

73. bronchial disorder.mp.

74. hyper-responsiveness wheez*.mp.

75. Respiratory Sounds/

76. lung function.mp.

77. ventilatory function.mp.

78. FEV.mp.

79. FEF.mp.

80. FVC.mp.

81. PEF.mp.

82. bronchial hyperreactivity.mp.

83. airway hyperreactivity.mp.

84. bronchial responsiveness.mp.

85. airway responsiveness.mp.

86. or/66-85

87. Rhinitis/

88. Rhinitis, Allergic, Perennial/

89. Rhinitis, Allergic, Seasonal/

90. hayfever.mp.

91. hay fever.mp.

92. fever, hay.mp.
93. seasonal allergic rhinitis.mp.

94. allergic rhinitides. $\mathrm{mp}$.

95. allergic rhinitis.mp.

96. rhiniti*.mp.

97. poll?nosis.mp.

98. pollenosis.mp.

99. Nasal Obstruction/

100. Conjunctivitis/

101. Conjunctivitis, Allergic/

102. conjunctivit*.mp.

103. rhino-conjunctivit*.mp.

104. or/87-103

105. Urticaria/

106. urticaria.mp.

107. Angioedema/

108. angioedema.mp.

109. or/105-108

110. Venom allergy.mp.

111. Drug Hypersensitivity/

112. drug alleregy.mp.

113. or/110-112

114. Anaphylaxis/

115. anaphylaxis react*.mp.

116. anaphylactic react*.mp.

117. anaphylactic shock*.mp.

118. anaphylactoid syndrome*.mp.

119. anaphylactoid react*.mp.

120. anaphylactic syndrome*.mp.

121. anaphylactoid shock*.mp.

122. acute systemic allergic react*.mp.

123. idiopathic anaphylaxis.mp.

124. systemic anaphylaxis.mp.

125. or/114-124

126. 44 or 51 or 58 or 65 or 86 or 104 or 109 or 113 or 125

127. 37 AND 126

\section{Available online at http://www.thepcrj.org}

